\title{
Comparing the accuracy of two electronic apex locators in the determination of working length and the detection of root perforations: An in vitro study
}

\author{
Kaveh Nasiri ${ }^{1 *}$ and Karl-Thomas Wrbas ${ }^{1,2}$ \\ ${ }^{1}$ Department of Endodontics, Center for Operative Dentistry and Periodontology, University of Dental Medicine and Oral Health, Danube Private University, \\ Krems, Austria \\ ${ }^{2}$ Department of Operative Dentistry and Periodontology, Center for Dental Medicine, Oral and Maxillofacial Surgery, Medical Center, University of Freiburg, \\ Freiburg i.Br., Germany
}

\begin{abstract}
Aim: To determine the accuracy of two electronic apex locators in measuring working length and detecting the location of root perforations.

Methodology: A total of 30 distal roots of extracted first maxillary molars were chosen. The actual working length was first measured. The electronic working length was obtained with Root ZX and Raypex 6. The differences between electronic working lengths and actual working length were calculated. Afterwards, the same roots were perforated artificially above the apical foramen. Both devices were utilized to locate perforations in the distal roots. Subsequently, the obtained measurements were compared. Intraoral environment was simulated employing a fixation device. Data were analysed using repeated measures ANOVA, paired sample t test, chi square, and Fisher exact tests. Statistical significance was considered at the 0.05 level.
\end{abstract}

Results: There was no significant difference among electronic working lengths, measured with the two devices, and actual working length ( $\mathrm{p}>0.05$ ). The percentage of accuracy of electronic working lengths, measured by Root ZX and Raypex 6, was $85.7 \%$ and $90.9 \%$ respectively within a tolerance of \pm 0.5 mm (p>0.05). Moreover, the difference between the devices was significant with regard to the location of root perforations $(\mathrm{p}<0.05)$.

Conclusions: Both electronic apex locators were able to determine canal length. Raypex 6 was more accurate than Root $\mathrm{ZX}$ in locating root perforations.

Abbreviations: AC: Apical constriction; AWL: Actual working length; DB: Distobuccal; EAL(s): Electronic apex locator(s); EWL(s): Electronic working length(s); WL: Working length.

\section{Introduction}

One of the most essential steps in effective root canal treatment is the determination of working length and the position of the apical constriction in the root canal [1]. Failure to measure the working length correctly leads to hindering satisfactory outcome in root canal treatment. There could be the following complications; over instrumentation or extrusion of the debris and filling material from the root canal and under instrumentation or under root filling [2]. Moreover, root perforations during access cavity and root canal instrumentation or inappropriate post space preparation may also complicate endodontic treatment [3]. These factors may increase the risk of periradicular inflammation and failure of treatment $[2,3]$.

Since the apical anatomy of each root canal is unique, accurate measurement of the working length is a challenge for the endodontic therapy [4]. The use of radiography methods for measurement might cause different complications such as distortion, shortening, and elongation. Furthermore, it is not always feasible to detect root perforation with radiographic techniques. To address this issue, it is suggested that electronic apex locators (EALs) can reduce the number of radiographs required. In fact, EALs supplement radiographic techniques for the determination of working length, and apical constriction as well as the detection of root perforation $[4,5]$.

Different EALs have been recommended for the determination of working length $[1,5,6]$. Root ZX is the most popular EAL which is based on the ratio type with dual Frequency $(8$ and $0.4 \mathrm{kHz})[1,5]$. Raypex 6 is the latest innovative EAL, which is based on the modification of fifth generation, in the Raypex series [6]. The purpose of this laboratory study was to measure the accuracy of Root ZX and Raypex 6 in determining the working length in comparison with the corresponding AWL. This study also aimed to detect the location of perforation site employing two different electronic devices.

\section{Material and method}

A total of thirty distobuccal roots of extracted first maxillary molars with single and straight root $\left(\leq 5^{\circ}\right)$ and mature apex were

${ }^{*}$ Correspondence to: Kaveh Nasiri, DDS, MSc (Endodontics), Department of Endodontics, Center for Operative Dentistry and Periodontology, University of Dental Medicine and Oral Health, Danube Private University, Krems, Austria, E-mail: DDS.Nasiri@web.de

Key words: accuracy, apex locator, perforation, working length

Received: April 20, 2019; Accepted: May 17, 2019; Published: May 21, 2019 

study

selected for this vitro study. The canal curvatures were obtained using Schneider's method [7]. It should be noted that roots with internal or external resorption, calcification and multi canals, verification of which was done through radiography evaluation and external examination, were excluded. The selected teeth were cleaned of soft, hard tissues, calculus, and debris. They were disinfected by immersion in $\mathrm{NaOCl}$ $5.25 \%$ for one hour. Afterwards, the samples were rinsed with distillate water and kept in $\mathrm{NaCl} 0.9 \%$ before use.

For simple identification, the teeth were randomly numbered from 1 to 30 . The crowns of each distobuccal root were sectioned at the cement-enamel junction with a diamond disc to provide unrestricted access to the canal and create a clear reference point. The canal orifices were located with DG16 explorer and negotiated with a k-file size 8. The pulp was extirpated with a barbed broach XXXXF (VDW, Munich, Germany). Afterwards, coronal enlargement was performed passively with Gates Glidden drill sizes 2-3. After each instrument, the canals were rinsed with $2 \mathrm{ml}$ of $\mathrm{NaOCl} 5.25 \%$.

\section{Actual working length in DB roots}

The actual working length (AWL) was measured by introducing a k-file size 10 (VDW, Munich, Germany) into the canal until the file tip was visible at the major apical foramen. This procedure was performed under a stereomicroscope at 20x magnification. Rubber stop was carefully adjusted to the coronal reference point. The file was withdrawn from the root canal and the distance between the file tip and rubber stop was determined with a digital measuring gauge (Bahag AG, Mannheim, Germany). Eventually, $0.5 \mathrm{~mm}$ was deducted from this length to obtain AWL. The patency was constantly checked for the canal with a size $8 \mathrm{k}$-file. The AWL is considered as the gold standard in comparison to EWLs. In order to set the apical constriction at 0.15 $\mathrm{mm}$, the preparation of all canals was performed with a k-file size 15 .

\section{Simulation of the clinical condition}

In order to simulate the clinical condition, a square plastic cup was used in this study. Four holes were made on the walls of the cup and four long screws were inserted into the holes and metal rings that were later used for the fixation of the samples. The pumice sponge was cut into small rectangular cubes in which the roots were placed and stabilized with the four long screws. Two-third of the sponge was immersed in $\mathrm{NaCl}$ 0.9\%.The metal lip clip was connected to the metal ring and the solution (Figure 1).

\section{Electronic working length in DB roots}

The electronic working length in distobuccal (DB) roots was measured with two different apex locators namely, Root ZX (J. Morita Mfg Corp., Kyoto, Japan) and Raypex 6 (VDW, Munich, Germany) according to the manufacturer's instructions. All samples were measured with both devices. The metal lip clip was placed in the cup and into the solution, indeed. Afterwards, the file clip of Root ZX was attached to a k-file size 15 and inserted into the DB canal apically until the EAL displayed a flashing bar in the middle of apex and 1 mark which indicated that the tip of the file has reached the prearranged length of the apical constriction. In the next step, the silicone stopper was carefully adjusted and the WL was recorded via digital measuring gauge. The same procedure was performed with Raypex 6 for the determination of DB root canals. However, k-file size 15 was introduced apically into the DB canal until the third green line was displayed. The EWLs were measured with both devices and then recorded. The differences between the EWLs and AWL were calculated. In the case that EWL was shorter than AWL, the value was negative. The value was considered positive

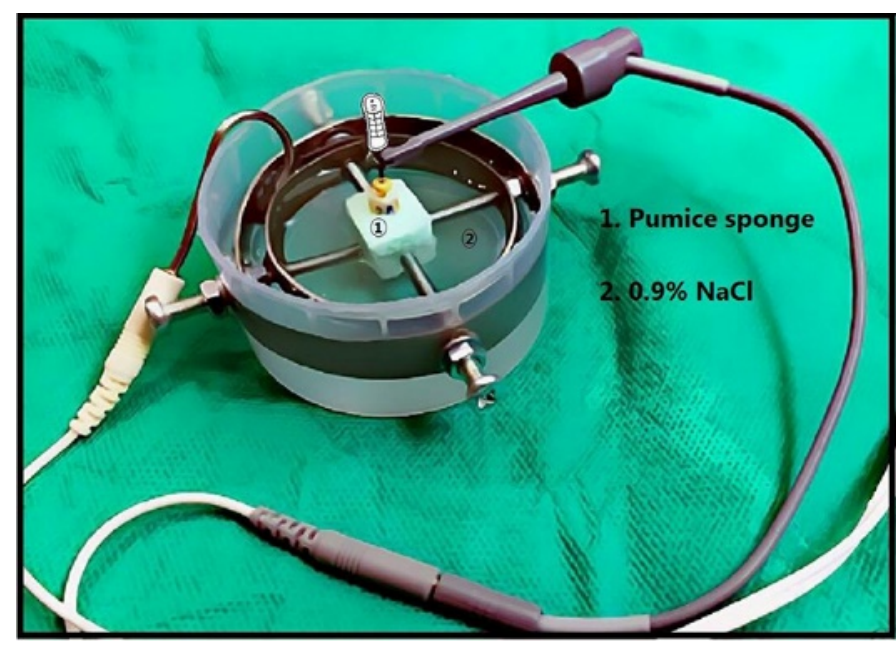

Figure 1. Fixation device showing the root placed in a pumice sponge and fixed with four long screws

when EWL was longer than AWL. Finally, the value was zero when the EWL and AWL were the same. The accuracy of the two devices was assessed within a tolerance limit of $\pm 0.5 \mathrm{~mm}$ to the AWL.

\section{Locating root perforations in $\mathrm{DB}$ roots}

To detect the location of the perforation site, Root ZX and Raypex 6 were used according to the manufacturer's instructions. Having determined the EWLs in the DB roots, a k-file size 17.5 (VDW, Munich, Germany) was held into the canal. Each sample was prudently fixed in the Mini Baby Vice. The perforation site was located with a digital measuring gauge and marked with a waterproof pen at $4 \mathrm{~mm}$ above the apical foramen. The groove was performed under $4.5 \mathrm{x}$ magnifying loupes (Zeiss, Jena, Germany) with a diamond needle bur fixed in highspeed with 45 degrees angulation to the root axis.

In the next step, the Gates Glidden drill sizes 2 and 1 were used in crown-down fashion until the file size 17.5 was observed in the perforation site. In other words, employing GGD size 1 , a hole 0.5 in diameter, was made in the inner root surface. To obtain the visual distance from the perforation site to the reference point, a k-file size 15 was inserted into the perforation site until the file tip was observed in the root surface under a stereomicroscope at $20 \mathrm{x}$ magnification (Bresser, Rhede, Germany). Rubber stop was warily adjusted to the coronal reference point. The file was then removed from the perforation site and the distance from the base of the stop to the file tip was measured with a digital measuring gauge.

The detection of root perforation in all samples was carried out employing both devices, Root ZX and Raypex 6. Each root was fixed in the fixation device (Figure 1) and to establish an electronic connection, the metal lip clip was immersed into the solution. The k-file size 15 was connected to a file clip of Root ZX and inserted into the root perforation site until the LED of the Root ZX displayed "APEX" which was signalized in a continuous audible tone. It was then withdrawn until the last green bar was shown on the LED. The k-file was then withdrawn completely from the artificial canal pathway and the electronic distance from the file tip to the rubber stop, which was indicated as the coronal reference point, was measured. To detect the location of perforation with Raypex 6, almost the same procedure was performed with the small difference that the file was inserted to the perforation site until the red dot was seen on the LED display and was then withdrawn until the LED showed the red bar. 

study

To determine the location of perforation in distal roots, the difference between electronic and visual lengths was calculated according to the following criteria: exact (zero value) was recorded when the file tip was located at the same level as the root surface, i.e. the electronic and visual lengths were the same; long (positive) was recorded when the file tip was beyond the perforation site; and short (negative) was recorded when the file tip was shorter than the perforation site. In fact, whether the electronic length was longer than the visual length or the opposite was the criterion based on which root perforations were detected.

In the current study, each measurement was performed three times and the mean values were calculated and reported. Schematic representation of the AWL- $0.5 \mathrm{~mm}$ and the position of the file tip in the root perforation were depicted in Figure 2.

\section{Statistical analysis}

All data obtained were recorded in an Excel sheet and analysed using SPSS software (IBM SPSS Statistics 21.0, IBM, Armonk, NY, USA). Repeated measures ANOVA was carried out to evaluate the actual length and the two electronic lengths measured with Root ZX and Raypex 6. In addition, paired sample t-test was run to assess the difference between EWLs and AWL as well as the distance from file tip to perforation site in the two devices. Chi square test was also used to evaluate the position of the file tip in different categorizations. Finally, Fischer exact test was used to assess the level of agreement between the two devices in each sample. The level of significance was set at $\alpha=0.05$ for all statistical tests.

\section{Results}

Table 1 illustrates the results of repeated measures ANOVA and paired sample t-test. There was no statistically significant difference between the AWL and EWLs using Root ZX and Raypex 6 ( $\mathrm{p}>0.05)$. According to Table 1, no statistically significant difference was observed between measurements made with Root ZX minus AWL and Raypex6 minus AWL.
Results of the distance from the file tip to the perforation site measured with two EALs are presented in Table 2. The results revealed that the average distance from the file tip to perforation site measured with Raypex 6 was shorter than the one measured with Root ZX. In other words, Raypex 6 identified the perforation site more accurately than Root ZX $(\mathrm{p}<0.05)$. The average distances from the file tip to the coronal reference point for visual and electronic detection of perforation with Root ZX and Raypex 6 were 9.510 \pm 0.79 , 9.642 \pm 0.81 , and $9.591 \pm 0.80$ respectively. The difference between the measurements is shown in Table 2 .

Based on the results of chi square test, presented in Table 3, no significant difference was found between the two devices in three categories: acceptable length, shorter than acceptable, and longer than acceptable within a tolerance of $\pm 0.5 \mathrm{~mm}$ in locating the file tip in AWL $(\mathrm{p}>0.05)$.

Table 4 presents the distribution of the file tip position in relation to the perforation site with two devices. Chi square test showed no statistically significant difference between Root ZX and Raypex 6 in the position of the file tip in different locations ( $p>0.05)$.

Fischer exact test was used (Table 5) to evaluate the level of agreement between the two devices for each sample. Regarding EWL's measurement, the results showed that 24 samples were in the range of the same acceptable length and two samples were shorter than the acceptable length. Based on the results of Fisher exact test, the level of agreement between the two devices associated with different categories of length was statistically significant $(\mathrm{p}<0.05)$.

Moreover, the level of agreement between the two devices was also measured for the location of the perforation site and the results revealed that in each of the 20 samples, the file tip was located over the perforation site and only in one sample, was the file tip located under the perforation site. However, no statistically significant difference was observed in the level of agreement between the two devices ( $p>0.05)$. In addition, the kappa value revealed that the accuracy of the agreement

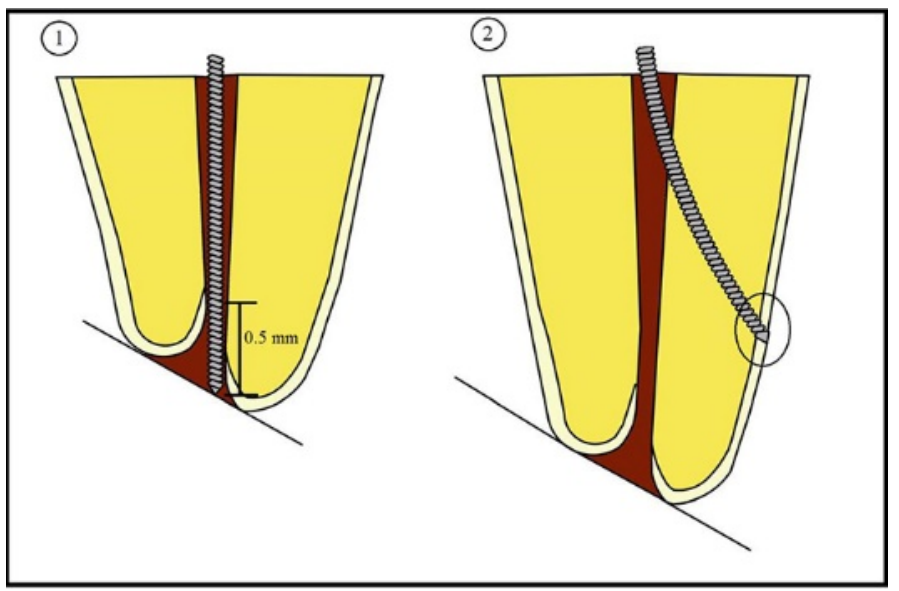

Figure 2. (1) Schematic of the position of the file tip in the AWL minus 0.5. (2) Schematic of the file tip position in the perforation site

Table 1. Mean and standard deviation ( $\mathrm{mm}$ ) of AWL, and EWLs and the difference between them

\begin{tabular}{|c|c|c|c|c|c|c|c|}
\hline \multirow{2}{*}{ Parameter } & \multicolumn{3}{|c|}{ Mean \pm SD } & \multirow{2}{*}{$\mathbf{P}^{*}$} & \multicolumn{2}{|c|}{ Difference between EWLs and AWL } & \multirow{2}{*}{$\mathbf{p}^{\dagger}$} \\
\hline & AWL & $\operatorname{Root} Z \mathbf{Z X}$ & Raypex 6 & & Root ZX- AWL & Raypex 6-AWL & \\
\hline Distance $(\mathrm{mm})$ & $13.009 \pm 0.91$ & $13.045 \pm 0.92$ & $13.007 \pm 0.93$ & 0.363 & $0.036 \pm 0.317$ & $-0.002 \pm 0.276$ & 0.354 \\
\hline
\end{tabular}

"Repeated measures ANOVA; $†$ Paired sample t-test 
Nasiri K (2019) Comparing the accuracy of two electronic apex locators in the determination of working length and the detection of root perforations: An in vitro study

Table 2. Mean and standard deviation of the distance from the file tip to the root perforation measured with Root ZX and Raypex 6 in mm

\begin{tabular}{|c|c|c|c|}
\hline \multirow{2}{*}{ Parameter } & \multicolumn{2}{|c|}{ Mean \pm SD } & Raypex 6 \\
\cline { 2 - 4 } & Root ZX & $0.081 \pm 0.067$ \\
\hline Distance from file tip to perforation site & $0.132 \pm 0.092$ & 0.001 \\
\hline
\end{tabular}

"Paired sample t-test.

Table 3. EWLs measurements in acceptable, shorter, and longer categories within a tolerance of $\pm 0.5 \mathrm{~mm}$ to the AWL

\begin{tabular}{|c|c|c|c|c|c|c|c|}
\hline \multirow[t]{2}{*}{ Device } & \multicolumn{2}{|c|}{$\begin{array}{c}\text { Acceptable } \\
\text { length }( \pm 0.5)\end{array}$} & \multicolumn{2}{|c|}{$\begin{array}{c}\text { Shorter than } \\
\text { Acceptable length }\end{array}$} & \multicolumn{2}{|c|}{$\begin{array}{c}\text { Longer than } \\
\text { acceptable length }\end{array}$} & $\mathbf{P}^{*}$ \\
\hline & (n) & $\%$ & (n) & $\%$ & (n) & $\%$ & \multirow{3}{*}{0.789} \\
\hline Root ZX & 25 & 85.7 & 3 & 8.6 & 2 & 5.7 & \\
\hline Raypex 6 & 27 & 90.9 & 2 & 6.1 & 1 & 3 & \\
\hline
\end{tabular}

"Chi square test.

Table 4.The position of the file tip in the perforation site after being measured with EALs

\begin{tabular}{|c|c|c|c|c|c|c|c|}
\hline $\begin{array}{l}\text { Distance from file tip position in } \\
\text { relation to the root perforation }(\mathrm{mm})\end{array}$ & \multicolumn{2}{|c|}{ Long (over the root surface $0.00<$ ) } & \multicolumn{2}{|c|}{ Exact (at the root surface $0.00=0.00$ ) } & \multicolumn{2}{|c|}{ Short (under the root surface $0.00>$ ) } & $\mathbf{P}^{\star}$ \\
\hline Device & (n) & $\%$ & (n) & $\%$ & (n) & $\%$ & \multirow{3}{*}{0.642} \\
\hline Root ZX & 24 & 80 & 4 & 13.3 & 2 & 6.7 & \\
\hline Raypex 6 & 25 & 83.3 & 2 & 6.7 & 3 & 10 & \\
\hline
\end{tabular}

"Chi square test.

Table 5. Accuracy level of agreement between Raypex 6 and Root ZX for each sample with regard to working length and perforation site

\begin{tabular}{|c|c|c|c|c|c|c|}
\hline \multirow{2}{*}{ Parameter } & & & \multicolumn{3}{|c|}{ Raypex 6} & \multirow{2}{*}{$\mathbf{P}^{*}$} \\
\hline & & & Acceptable & Shorter & Longer & \\
\hline \multirow{4}{*}{ Working length } & \multirow{3}{*}{ Root ZX } & Acceptable ${ }^{A}$ & 24 & 0 & 1 & \multirow{3}{*}{0.015} \\
\hline & & Shorter ${ }^{\mathrm{B}}$ & 1 & 2 & 0 & \\
\hline & & Longer $^{\mathrm{C}}$ & 2 & 0 & 0 & \\
\hline & & & Long & Exact & Short & \multirow{4}{*}{0.194} \\
\hline \multirow{3}{*}{ Perforation site } & \multirow{3}{*}{ Root ZX } & Long $^{\mathrm{D}}$ & 20 & 3 & 1 & \\
\hline & & Exact $^{\mathrm{E}}$ & 3 & 0 & 1 & \\
\hline & & Short $^{\mathrm{F}}$ & 1 & 0 & 1 & \\
\hline
\end{tabular}

"Fisher's exact test; A: Acceptable length \pm 0.5 ; B: Shorter than -0.5 ; C: Longer than +0.5 ; D: Over the root surface $0.00<$; E: At the root surface $0.00=0.00$; F: under the root surface $0.00>$.

between the two devices in the variable working lengths (kappa=0.447) was higher than that of the two devices for the determination of perforation site in root canals (kappa $=0.118)$

\section{Discussion}

In root canal treatment, the apical limit for instrumentation and filling of root canals is the apical constriction (AC). On this account, detecting the precise location of the apical constriction is undoubtedly an important goal in endodontic treatment [8]. Furthermore, one of the unpredictable complications in root canal therapy is the root perforation. The opportune detection and treatment of root perforation is essential for successful prognosis and treatment [9]. The goal of the current study was to assess two different apex locators in determining the working length. In addition, this study aimed to detect the location of root perforation along the root surface.

To determine the working length as well as to detect the location of root perforation site, thirty samples were used in this study. Several in vitro and in vivo studies evaluated the accuracy of different EALs in measuring $\mathrm{WL}$ and locating root perforations [8-10]. According to the literature, examining the efficacy of different EALs in laboratory environment to measure the $\mathrm{WL}$ and to locate root perforations has been rarely carried out in one study, which is, thus, one of the strengths of this study [11].

The accuracy of results, whether in comparing different EALs to determine the WL or in detecting root perforations, is only achievable when each sample is evaluated by all devices $[5,8]$. That is why all roots were assessed with both Root ZX and Raypex 6 in this study.

As reported in previous studies, AWL was recorded $0.5 \mathrm{~mm}$ shorter than the major foramen [12]. In addition, Plotino et al. [13] reported that the distance between the apical constriction and apical foramen was located within $0.5 \mathrm{~mm}$. Therefore, in the current study, to obtain the actual working length, $0.5 \mathrm{~mm}$ was subtracted from the measurement. In various studies, the acceptable tolerance limit was adjusted to \pm 0.5 $\mathrm{mm}$ of the apical constriction $[5,13,14]$. Researchers also stated that measurement within this tolerance is impeccable and is, therefore, clinically acceptable. Consequently, in the present investigation, tolerance limit was set within $\pm 0.5 \mathrm{~mm}$ of the actual working length.

The three arrangements: the acceptable length within $\pm 0.5 \mathrm{~mm}$, shorter than $-0.5 \mathrm{~mm}$, and longer than $+0.5 \mathrm{~mm}$ were used as the criteria to evaluate the accuracy of the two devices in the determination of the working length in comparison to the actual working length as done in the study by Bahrololoomi et al. [15]. Furthermore, three categories: long, exact, and short were selected for the evaluation of the position of the file tip to the perforation site following the study by Kaufman et al. [16].

Following the procedure of similar studies, to decrease the procedural measurement errors, all measurements were made three times and the averages were calculated and recorded in Excel. The devices were always fully charged while measuring and the saline was changed after measuring each sample [12,17]. 

study

Different electro conductive materials including agar [15], alginate [16], gelatine [18], and saline [19] have been utilized to simulate a clinical situation in various studies. These studies reported that since apex locator's function is more according to electrical principles than the biological features of intraoral tissues, the samples must be put in an electro conductive material. On this account, in this study, normal saline was poured into the square plastic cup to simulate the oral environment. The samples were put in a piece of pumice sponge which was mounted on a metal ring with four long screws. This fixation device is simple and economic. Moreover, the clinician cannot observe the apex root or the perforation site. The fixation device is made as a combination of two different devices used in two vitro studies $[19,20]$.

Based on the findings of the previous studies, the success rate of EALs in determining the WL decreases with the increase of the apical diameter [10]. Hence, in the present study, a k-file size 15 , which is the ideal size to determine electronic working length, was utilized as employed by Jadhav et al. [6].

Examining the efficacy of two EALs in determining the working length showed that both devices are able to determine the working length in the simulation environment and no statistically significant difference was found between the measurements. This result is consistent with other studies $[8,10]$. Regarding locating root perforations with the two devices, the distance from the file tip to perforation site, which was measured with Rypex 6 , was shorter than the one measured with Root ZX and the difference was statistically significant. In other words, Raypex 6 detected the perforation site more accurately than Root ZX. Our results coincided with Haupt and Hulsmann study [11] in which Raypex 6 has showed the most accurate device.

Lastly, in the course of this study, kappa value showed that the reliability of the two devices in determining working lengths is more accurate than in the detection of root perforations.

\section{Conclusions}

Under the conditions of this vitro study, both devices showed similar accuracy in determining the working length. Raypex 6 was found as more accurate than Root ZX in locating root perforation. Thus, based on the findings of this study, it can be concluded that both devices tested, are acceptable clinical tools for determining the working length. In the case of root perforation, Raypex 6 brings more precise results in comparison to Root ZX and is very helpful for the special endodontic therapy.

\section{References}

1. Vanitha S, Sherwood IA (2019) Comparison of three different apex locators in determining the working length of mandibular first molar teeth with irreversible pulpitis compared with an intraoral periapical radiograph: A block randomized, controlled, clinical trial. J Investig Clin Dent 10: e12408. [Crossref]

2. Chaudhary S, Gharti A, Adhikari B (2018) An in vivo comparison of accuracy of two electronic apex locators in determining working length using stainless steel and nickel titanium files. Clin Cosmet Investig Dent 10: 75-82. [Crossref]
3. Marroquín BB, Fernández CC, Schmidtmann I, Willershausen B, Goldberg F (2014) Accuracy of electronic apex locators to detect root canal perforations with inserted metallic posts: an ex vivo study. Head Face Med 10: 57. [Crossref]

4. Rambabu T, Srikanth V, Sajjan GS, Ganguru S, Gayatri C, et al. (2018) Comparison of tentative radiographic working length with and without grid versus electronic apex locator. Contemp Clin Dent 9: 88-91. [Crossref]

5. Wrbas KT, Ziegler AA, Altenburger MJ, Schirrmeister JF (2007) In vivo comparison of working length determination with two electronic apex locators. Int Endod J 40: 133-138. [Crossref]

6. Jadhav GR, Mittal P, Patil V, Kandekar P, Kulkarni A, et al. (2018) Accuracy of different apex locators in teeth with simulated apical root resorption: An in vitro study. Folia Med (Plovdiv) 60: 624-631. [Crossref]

7. Schneider SW (1971) A comparison of canal preparations in straight and curved root canals. Oral Surg Oral Med Oral Pathol 32: 271-275. [Crossref]

8. Moscoso S, Pineda K, Basilio J, Alvarado C, Roig M, et al (2014) Evaluation of Dentaport ZX and Raypex 6 electronic apex locators: an in vivo study. Med Oral Pato Oral Cir Bucal 19: e202-e205. [Crossref]

9. Nazari Moghaddam K, Nazari S, Shakeri L, Honardar K, Mirmotalebi F (2010) In vitro detection of simulated apical root perforation with two electronic apex locators. Iran Endod J 5: 23-26. [Crossref]

10. Aydin U, Karataslioglu E, Aksoy F, Yildirim C (2015) In vitro evaluation of Root ZX and Raypex 6 in teeth with different apical diameters. J Conserv Dent 18: 66-69. [Crossref]

11. Haupt F, Hülsmann M (2018) Consistency of electronic measurements of endodontic working length when using multiple devices from the same manufacturer-an in vitro study. Clin Oral Investig 22: 3107-3112. [Crossref]

12. Saatchi M, Iravani S, Akhavan Khaleghi M, Mortaheb A (2017) Influence of root canal curvature on the accuracy of root ZX electronic foramen locator: An in vitro study. Iran Endod J 12: 173-178. [Crossref]

13. Plotino G, Grande NM, Brigante L, Lesti B, Somma F (2006) Ex vivo accuracy of three electronic apex locators: Root ZX, elements diagnostic unit and apex locator and ProPex. Int Endod J 39: 408-414. [Crossref]

14. Santhosh L, Raiththa P, Aswathanarayana S, Panchajanya S, Reddy JT, et al. (2014) Influence of root canal curvature on the accuracy of an electronic apex locator: An in vitro study. J Conserv Dent 17: 583-586. [Crossref]

15. Bahrololoomi Z, Soleymani AA, Modaresi J, Imanian M, Lotfian M (2015) Accuracy of an electronic apex locator for working length determination in primary anterior teeth. $J$ Dent (Tehran) 12: 243-248. [Crossref]

16. Kaufman AY, Fuss Z, Keila S, Waxenberg S (1997) Reliability of different electronic apex locators to detect root perforations in vitro. Int Endod J 30: 403-407. [Crossref]

17. Connert T, Judenhofer MS, Hülber-J M, Schell S, Mannheim JG, et al. (2018) Evaluation of the accuracy of nine electronic apex locators by using Micro-CT. Int Endod J 5: 223-232. [Crossref]

18. Khattak O, Raidullah E, Francis ML (2014) A comparative assessment of the accuracy of electronic apex locator (Root ZX) in the presence of commonly used irrigating solutions. J Clin Exp Dent 6: e41-e46. [Crossref]

19. Chakravarthy Pishipati KV (2013) An in vitro comparison of Propex II apex locator to standard radiographic method. Iran Endod J 8: 114-117. [Crossref]

20. Christofzik DW, Bartols A, Khaled M, Größner-Schreiber B, Dörfer CE (2017) The accuracy of the auto-stop function of different endodontic devices in detecting the apical constriction. BMC Oral Health 17: 141. [Crossref]

Copyright: (C)2019 Nasiri K. This is an open-access article distributed under the terms of the Creative Commons Attribution License, which permits unrestricted use, distribution, and reproduction in any medium, provided the original author and source are credited. 